\title{
L'UTILISATION DES DÉBROUSSAILLANTS CHIMIQUES DANS LA DESTRUCTION DES TAILLIS DE CHARME
}

\section{$\mathrm{I}^{\circ}$ Les buts poursuivis}

Dans le département de la Nièvre comme ailleurs, la conversion des taillis-sous-futaie en futaie régulière a connu des vicissitudes diverses depuis 1828 , date de la première réalisation sur 500 ha. A part 3000 ha des Bertranges mis en conversion en 1886 non sans. d'âpres discussions entre le Conseil d'Administration et le Service local, farouche tenant du taillis-sous-futaie, on peut dire que l'idée de la conversion généralisée n'a pris corps dans la Nièvre qu'en I.924 sous l'influence de M. Turc, alors Inspecteur Adjoint à Cosne. Les premières mises en vieillissement des taillis, à l'exception de 3000 hectares des Bertranges, datent de 1926. Encore faut-il ajouter qu'une fraction des massifs aménagés en conversion était maintenue sous le régime du taillis pour satisfaire aux conceptions classiques re la conversion.

C'est dire que les traitement divers groupés sous le vocable commode de Taillis-sous-Futaie ont laissé une marque profoncle dans les peuplements domaniaux nivernais.

Cependant, les martelages du type ancien (30 baliveaux, Io à I 5 modernes, I ancien) ne laissant sur les taillis destinés à l'alimentation des forges que des " étalons ) en nombre réduit ont été poursuivis assez lontemps, pratiquement jusqu'en r886; les balivages intensifs ont été pratiqués à leur densité maximum assez tardivement (I902); si bien que les peuplements domaniaux de la Nièvre se trouvent encore très riches en réserves et comportent presque tous une futaie suffisante pour assurer la régénération.

Le charme a envahi les recrus dans toutes les stations de pédonculés, dans les vallons et sur tous les versants frais et a cheminé peu ou prou sur tout le plateau où il participe presque toujours pour une part importante à la formation des taillis et des sousétages.

Pour exploiter l'échelonnement assez limité des âges des réserves des taillis-sous-futaie, il a fallu procéder à des régénération directes 
dans les ( peuplements limites ), c'est-à-dire ceux où la futaie est à effectif suffisant pour assurer la régénération et où le taillis n'offre plus aucune ressource pour la renouveler.

Le charme s'est tout de suite révélé comme l'ennemi $1^{\circ}$ I de la régénération et sa destruction a été depuis 1925, date de la première régénération directe, une préoccupation constante pour le Service local.

'Tous les procédés culturaux de destruction ou au moins de nentralisation ont été essayés depuis cette époque avec des fortunes diverses. Les techniques à long terme sont bien au point, mais le problème de la destruction immédiate n'a été résolı que dans les cas très particuliers et limités, où l'extraction pure et simple peut être réalisée sans frais par des prestataires et au moment voulu. II fallait donc chercher autre chose.

\section{$2^{\circ}$ Les premiers essais de débrousaillants chimiques sur placettes}

Depuis quelques années, nous avions suivi de près le développement en agriculture du désherbage et du débroussaillement par ( hormones synthétiques ) et nous avions pensé à utiliser ces produits ou des produits similaires pour la destruction du charme.

Diverses Sociétés, saisies du problème nous ont adressé des échantillons de leurs produits habituels pour essais, mais les buts recherchés par l'agrriculture, les modes et les conditions d'emploi étaient inapplicables en forêt; une étude particulière était nécessaire.

Je hasard de relations personnelles nous ayant mis en contact avec des Ingénieurs d'une Société de Produits Chimiques, nous leur avons exposé la question et ils décidèrent de s'y attaquer.

Les premiers essais d'emploi de débroussaillants chimiques avec comme objectif précis: la destruction du charme, ont eu lieu dans l'inspection de Nevers-Nord en juin I95I.

Nous résumerons très rapidement les premiers essais:

6 produits différents ayant été mis au point par la Société, ils ont été essayés à des concentrations variables, aussi bien par badigeon de souche que par aspersion, sur des souches de section plus ou moins récente, sur des semis et rejets d'âges divers, à des époques variables. En bref, plus de 200 placettes étaient en fonctionnement.

Les premières constatations des résultats, faites en 1952 et 1953 , ont amené les conclusions suivantes:

$I^{\circ}$ Un seul produit a été retenu. Il est devenu le " débroussaillant PROCIDA ) miscible à l'eau en vente jusqu'en I955 (I1 a été ensuite modifié à nouveau pour accroître son efficacité).

$2^{\circ}$ L'aspersion dont les essais avaient été faits sur des rejets trop âgés a tout d'abord été abandonnée. 
$3^{\circ}$ L'application sur souche s'est avérée efficace à des doses variables en fonction de la date de l'exploitation et de l'application.

$4^{\circ}$ Il apparaissait certain que la destruction par badigeon sur souches exploitées depuis 8 jours à un mois aux doses de 5 à ro $\%$ était totale (Il faut noter pour la suite que les essais sur souche avaient été faits en octobre I95I).

\section{$3^{\circ}$ Les premiers essais sur une coupe de taillis}

En I953, il semblait bien, que le but était près d'être atteint, mais. il restait à transporter les opérations sur un plan qu'on peut qualifier d' " industriel ». L'emploi en grande série sur des coupes exploitées très irrégulièrement pouvait donner des résultats très différents de l'emploi sur place d'essai par des Agents Techniques soigneux et appliqués. Il fallait en outre connaitre le prix de revient des opérations et confirmer les premiers calculs faits sur les placettes d'essais.

Au printemps I953, nous tentions un essai général sur la

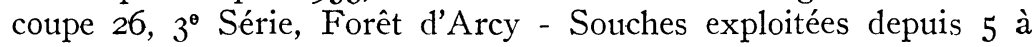
Io mois.

Exploitation: Automne I952 - Badigeon: de mai à août I953.

Concentrations : (DP) $7 \%$ et Io \% (DP $=$ Débroussaillant Procida).

Un lot témoin sur I hectare.

Consommation: 9,21 pour 6 ha 60 .

Cet essai relativement limité sur vieilles souches, était destiné précisément à constater les résultats dans les plus mauvaises conditions.

A l'automne I953, les rejets sont courbés et jaunissants et se dessèchent très brusquement.

Les échantillons prélevés au cours de l'hiver montrent la base des rejets gonflée et recouverte sur une hauteur variable d'un rhytidome liégeux, épais, profondément fissuré et nécrosé.

Au printemps I954, le tiers des souches est mort, le reste rejette à côté des rejets desséchés de l'année précédente encore sur la souche.

Au cours de l'été et de l'automne I954, les nouveaux rejets sont visiblement atteints de la même déformation que leurs prédécesseurs. Certaines souches ont des rejets traînants. La mortalité continue, mais lentement. La hauteur du charme dans la zone traitée est inférieure de moitié à celle de la zone témoin.

(En I954, les premiers essais au pulvérisateur ont eu lieu dans cette parcelle). 


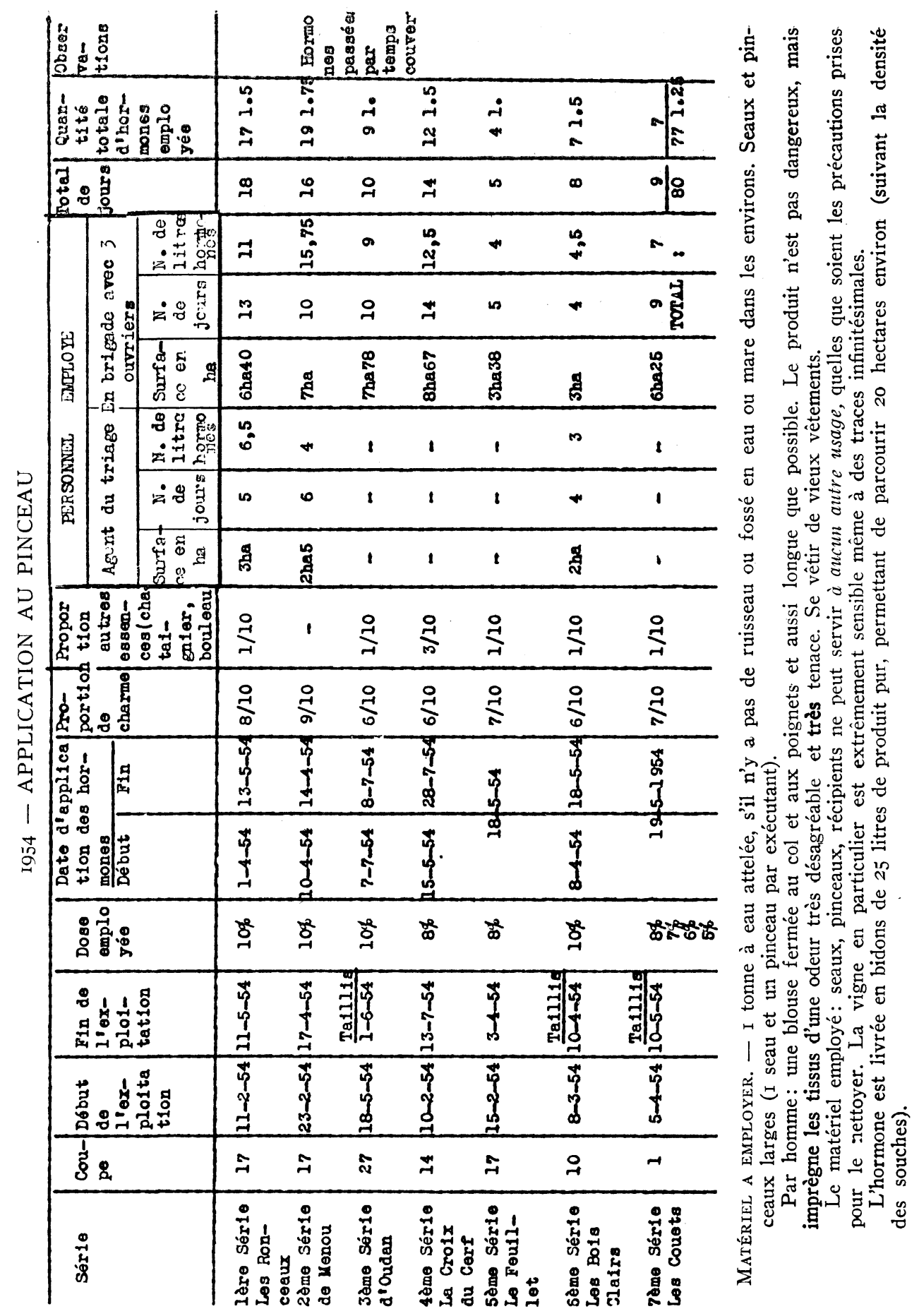




\section{$4^{\circ}$ Les premiers essais en grande série}

Au printemps 1954, un essai général est tenté dans des conditions normales, c'est-à-dire en s'efforçant de suivre les exploitations dans la mesure où le service des agents locaux le permettait.

Le tableau ci-joint résume les opérations.

Le prix de revient s'établissait comme stit en 1954 :

Nombre de journées de travail : 80 à i $200 \ldots \ldots \ldots .96000 \mathrm{~F}$ Nombre de litres de débroussaillant : 771.25 à $906 \ldots 69989 \mathrm{~F}$ Transport d'eau par tonne ................ $4000 \mathrm{~F}$ Amortissement du matériel (largement calculé) .... $8000 \mathrm{~F}$

Soit pour 53 ha $(52,98)$.

I77980 F

Prix de revient à l'ha: $3358 \mathrm{~F}$.

Les résultats. - Cette expérience nous a d'abord surpris par des résultats assez décevants, la plupart des souches ont rejeté normalement ou presque, la première et la deuxième années, alors que des opérations d'application au pinceau prescrites aux adjudicataires par les conditions particulières de vente de certaines coupes dès l'automne I954 donnaient des succès spectaculaires et définitifs avec les mêmes doses employées par des ouvriers payés par l'exploitant, c'est-à-dire bien plus difficiles à surveiller.

La seule explication était dans les dates d'application:

l'opération faite sur les coupes de la forêt d'Arcy se situait entre le $\mathrm{I}^{\text {er }}$ avril et le 7 juillet 1954 , c'est-à-dire au cours de la montée de la sève,

les opérations imposées aux adjudicataires avaient été rigoureusement surveillées et exécutées à l'automne aussitôt après l'exploitation, c'est-à-dire au retour de sève.

Et c'est à l'automne que les premières expériences d'application sur souche avaient été faites cn forêt des Bertranges.

Contrairement à ce qui avait été affirmé par les Ingénieurs de PRocina: savoir que tous les mouvements de sève étaient favorables à l'application du produit, il apparaissait certain que la montée de sève rejetait le produit vers l'extérieur et que la période de départ de la végétation était la plus mauvaise pour l'application. A tel point que les applications faites au mois de juillet paraissaient plus efficaces que celles faites au mois d'avril et mai. 


\section{$5^{\circ}$ Les constatations en 1956 sur les placettes d'essai de 1951}

Dans le même temps où se déroulaient ces opérations à Arcy, les placettes d'essais de I95 I en forêt des Bertranges se couvraient d'une végétation exubérante de tremble et morts-bois (Nous avons choisi des places sans semenciers' d'essence chêne) et parmi eux nous constations d'année en année que les charmes traités avec les doses faibles en I95I continuaient à se dessécher. C'est ainsi qu'en I956 nous avons observé la mort de rejets de charmes mesurant plus de deux mètres de hauteur.

Le fait mérite d'être signalé, car nous soupçonnons que dans l'avenir l'emploi de doses bien étudiées pourra permettre d'abriter dans les taillis de charme de jeunes plants d'essences d'ombre ou de demiombre sans qu’il soit nécessaire ultérieurement à les dégager.

\section{$6^{\circ}$ La reprise des essais de pulvérisation}

Dès l'instant où il était établi que la montée de sève était défavorable à l'application du débroussaillement chimique, il était nécessaire de trouver une autre solution. L'exploitation des coupes se déroulant normalement de l'automne au printemps, une grande partie de la surface traitée échapperait à l'effet du débroussaillant.

Nous avons donc pensé à reprendre la pulvérisation: sur des bases nouvelles.

Le fabricant avait perfectionné son débroussaillant en I955 et le présentait sous une forme très concentrée appelée $\mathrm{P} 80$. Nous avons essayé ce nouveau produit par pulvérisation pour l'ouverture de lignes d'aménagement en terrain difficile.

Les conditions d'emploi ont été les suivantes:

- Exploitation de la ligne ouverte par des prestataires ou des exploitants suivant les cas,

- Traitement de rejets de deux ans rabattus au goujard à $30 \mathrm{~cm}$ environ pour ne pas consommer trop de liquide.

- Traitement de rejets d'un an de 25 à $30 \mathrm{~cm}$.

Les résultats ont été excellents sur rejets d'un an; moins spectaculaires sur les rejets de deux ans rabattus; un certain nombre rle souches ont en effet rejeté une seconde fois et un deuxième traitement à demi-dose a été nécessaire.

En terrain incommode imposant l'emploi du pulvérisateur à dos et où toutes les souches de toutes essences devaient être traitées dans un taillis vigoureux âgé de 2 ans rabattu au goujard, le prix de revient d'une opération se monte environ à 20 ou $25000 \mathrm{~F}$ par ha. Si on doit passer" une seconde fois sur les rejets suivants, le prix atteint 30 à $35000 \mathrm{~F}$ à l'ha. La consommation d'eau est cle 4 à 500 litres ì l'ha à $0,75 \%$ de $\mathrm{P} 80$. 







\section{$7^{\circ}$ Les essais en 1955}

La mème année, nous avons continué les deux systèmes en forêt d'Arcy.

Comme en 1954, toutes les coupes exploitées ont été traitées au pinceau. Le tableau précédent résume les opérations.

Le prix de revient a été le suivant:

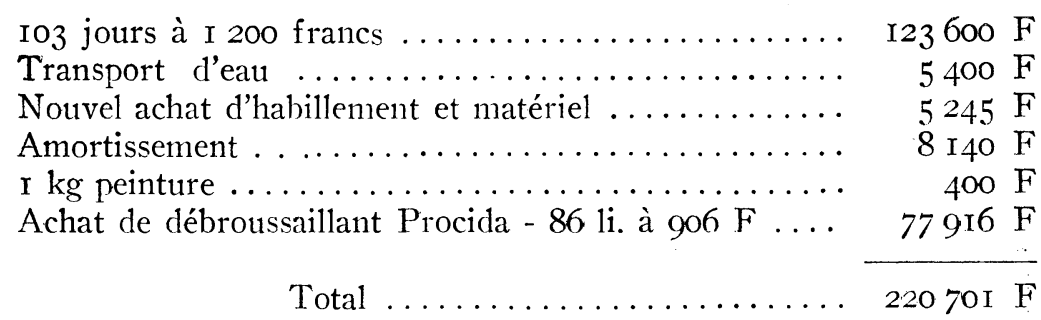

Surface traitée: 49 ha 24, soit à l'ha: $4480 \mathrm{~F}$.

Une partie des coupes des années précédentes déjà traitées par application sur souche a été traitée au pulvériseur sur rejets d'âges variables en partie avec l'ancien débroussaillant Procida en partie avec le nouveau débroussaillant $\mathrm{P} 8 \mathrm{o}$.

Le tableau ci-joint résume les opérations:

Le prix de revient s'établit comme suit:

39 journées 5 à I $200 \mathrm{~F}$ (C.S. non comprises) .... $47400 \mathrm{~F}$

Charges Sociales ................. $9000 \mathrm{~F}$

Transport eau ................... $2000 \mathrm{~F}$

Hormones: I9 1.75 à $906 \mathrm{~F} \ldots \ldots \ldots \ldots \ldots \ldots \ldots$. $\ldots \ldots 93$ F 50

o 1.75 à $4000 \mathrm{~F} \ldots \ldots \ldots \ldots \ldots \ldots$. $3000 \mathrm{~F}$

Surface traitée: 25 ha 7 .

79293 F 50

Prix à l'ha: $3085 \mathrm{~F}$

auquel il faut ajouter une partie de l'amortissement du matériel déjà compté lors de l'application au pinceau.

Le prix de revient de la pulvérisation en 1955 peut être estimé à $3500 \mathrm{~F}$ l'ha en terrain normal.

Les résultats ont été variables suivant l'âge des rejets, le produit employé et les doses d'emploi, mais il apparaissait clairement que la pulvérisation du $\mathrm{P} 80$ à $0,3 \%$ était efficace, alors que la dose normale est de 0,8 à I \%. 







\section{$8^{\circ}$ Les conclusions au début de 1956}

Tous ces essais un peu heurtés troublés par des difficultés de crédits et le changement de concentration et de nature du produit employé ont au moins permis, si j'ose cette expression, de débroussailler le problème. Une ligne de conduite assez ferme a pu être dégagée pour la poursuite des opérations en 1956 et 1957 .

Au début de 1956 , les conclusions à tirer des essais précédents sont les suivantes:

$\mathrm{I}^{\circ}$ L'application par badigeon de $\mathrm{P} 80$ mêlé à l'eau à la dose de I \% sur souche d'exploitation récente est d'une efficacité totale, sous réserve de l'exécuter entre le $\mathrm{I}^{\text {er }}$ septembre et le I $_{5}$ février, c'est-à-dire à l'exclusion des périodes de poussée de sève.

L'époque la plus favorable reste toujours la période de descente de sève, c'est-à-dire octobre-novembre.

$2^{\circ}$ Même si, à concentration plus faible, les résultats de l'application au pinceau ne sont pas spectaculaires dès la première saison de végétation, on constate que la mortalité continue pendant très longtemps.

$3^{\circ}$ Le prix de revient de l'application au pinceau est de l'ordre de $4000 \mathrm{~F}$ par ha.

$4^{\circ} \mathrm{La}$ pulvérisation de $\mathrm{P} 80$ à I $\%$ dans l'eau est très efficace sur rejets de 20 à $30 \mathrm{~cm}$ de hauteur.

Ceci limite la période d'application à juillet-août.

Il semble bien que non seulement le rejet, mais la souche, soit détruite.

En tous cas, la dose de I \% est une dose maxima qui n'est à atteindre que pour les rejets de charme. Il est préférable pour la réussite de mouiller davantage avec une concentration plus faible. Il vaut mieux, sur une même surface, utiliser Ioo litres à $0,50 \%$ que 50 litres à I \%, tout dépend de la plus ou moins grande facilité d'approvisionnement en eau et du prix de la main-d'œuvre.

$5^{\circ}$ Une constatation intéressante: le châtaignier assez résistant au traitement sur souche est très sensible à la putlvérisation même à doses très faibles $(0,3 \%)$.

\section{$9^{\circ}$ Les essais en 1956 et les incidents d'emploi}

En I956, nous devions combiner les deux systèmes:

22 ha de taillis exploité ont été traités au pinceau à la dose de I \%, du 5 décembre I955 au Io juin 1956, pour déterminer le degré exact d'efficacité dı $\mathrm{P} 80$.

I9 ha de taillis ont été traités par pulvérisation sur rejets du 28 juin au $3 \mathrm{I}$ août 1956 à des doses de 0,5 et $\mathrm{I} \%$.

J.es deux tableaux ci-joints résıment les opérations:

Les résultats seront constatés en 1957. 


\section{I956 - APPLICATION AU PINCEAU}

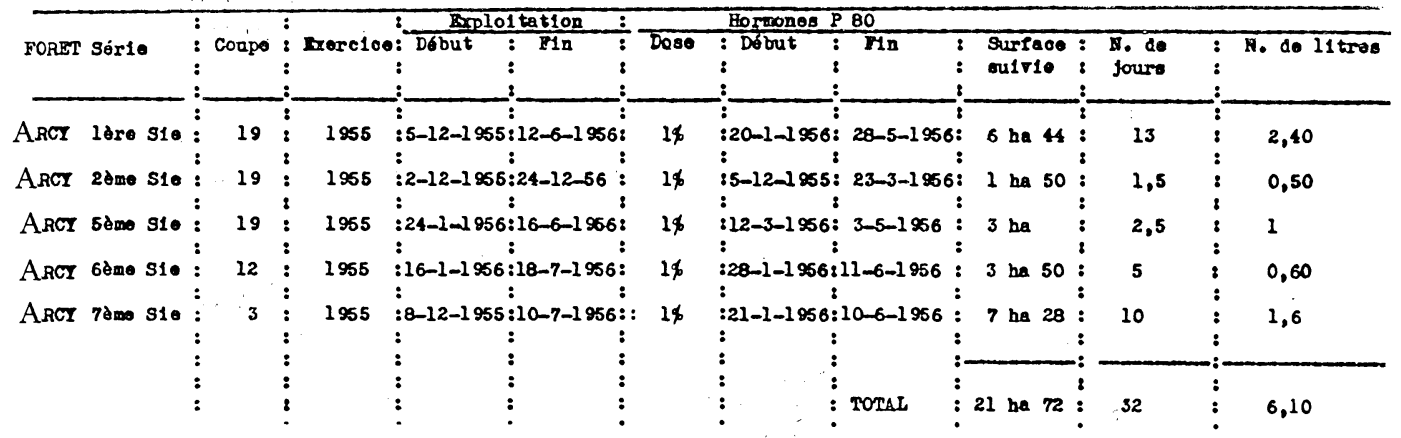

Observations. - Ce travail a été effectué en totalité par les Agents MorReau, Levin et Cauchy.

Le prix de revient à l'ha serait le suivant si ce travail avait été exécuté par des ouvriers:

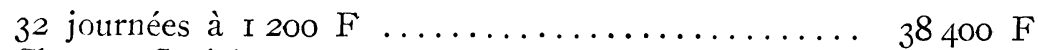

Charges Sociales ................. 7 I 37 F

Hormones : 61 io à $4000 \mathrm{~F} \ldots \ldots \ldots \ldots \ldots \ldots .24400 \mathrm{~F}$

Surface traitée: $2 \mathrm{I}$ ha 72 .

$69937 \mathrm{~F}$

Prix de revient à l'ha: $3220 \mathrm{~F}$

plus amortissement du matériel.

I956 - PULVERISATION

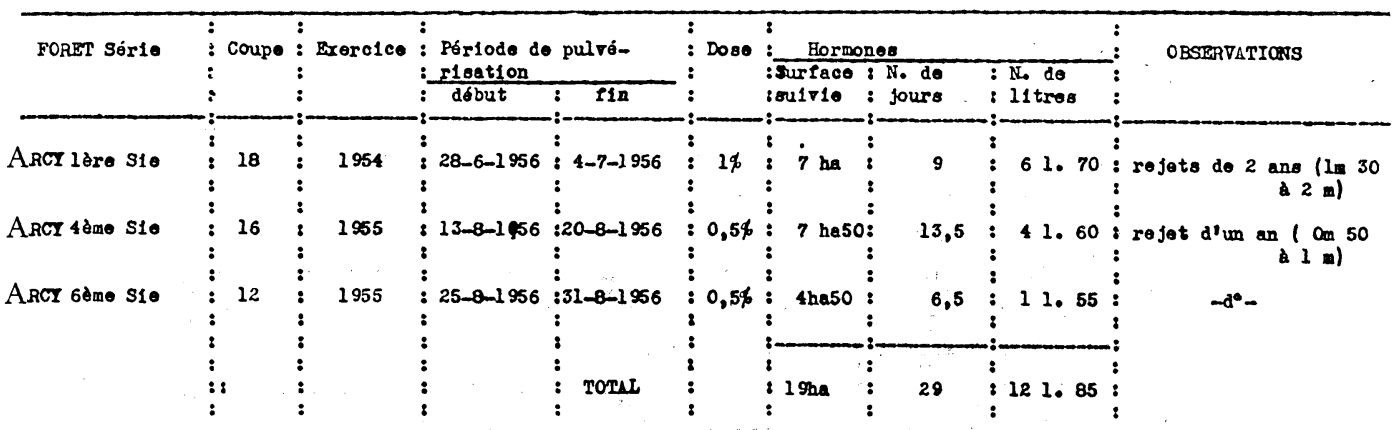

Notre intention était de traiter également par pulvérisation les rejets rescapés de l'application au pinceau, une partie dés opérations ayant été effectuée à la montée ‘de sève. 
Malheureusement, outre le manque de crédits, des incidents assez sérieux nous ont obligé à suspendre les opérations de pulvérisation.

Aussi bien en forêt des Bertranges où continuaient les ouvertures de lignes qu'en forêt d'Arcy où étaient faits les essais sur taillis, des intoxications plus ou moins graves ont été constatées avec des malaises qui ont duré plus ou moins longtemps: battements de cœur, jambes molles, manque d'appétit, fièvre, sécrétion lacrymale avec sensation de brûlure.

Nous avons immédiatement avisé le fabricant du produit dont les représentants ont manifesté un étonnement qui paraissait sincère car ils n'avaient jamais. eu l'occasion, parait-il, de constater de tels inconvénients dans le personnel de l'usine productrice.

Il semble bien que les conditions d'emploi soient à surveiller de très près: à l'usine, le produit n'est pas pulvérisé dans l'atmosphère.

Nous avons l'intention d'étudier cette affaire de près en 1957.

Les essais sur des rejets déjà élevés (et notamment sur châtaignier) avaient obligé à tenir les lances en hauteur. Un certain nombre de précautions doivent donc être prises.

\section{IO ${ }^{\circ}$ Premier essai de dégagement sélectif des plantations résineuses}

Au cours de l'été 1956, un premier essai du P. 8o à faible dose a été tenté sur une surface appréciable ( I ha environ) de plantation résineuse dans un contrat F.F.N. clépendant de l'Inspection de $\mathrm{Ne}$ vers-Sud.

Le résultat 'semble satisfaisant, mais il faut attendre pour apprécier les effets réels, la saison de végétation 1957.

\section{La situation au début de 1957}

Les traitements à mettre en œuvre en vue de la destruction du charme dans, les taillis sont au point:

$I^{\circ}$ Application de $\mathrm{P} 80$ à I \% dans l'eau sur les souches après l'exploitation en 'lehors des périodes de montée de sève.

$2^{\circ}$ Pulvérisation sur les rejets d'un an longs de 20 à $30 \mathrm{~cm}$ des souches non traitées au pinceau en raison de leur exploitation tardive avec concentration de 0,5 à I $\%$ suivant les quantités d'eau dont on dispose. La consommation d'eau étant à I \% d'environ 80 litres par ha sur un taillis à $7 /$ Io de charme.

$3^{\circ}$ Pulvériser en même temps que les rejets des souches non traitées au pinceau les rejets rescapés des souches traitées.

En fait, la seule application au pinceau bien conduite assure des résultats très suffisants pour le but à atteindre.

Depuis trois ans, la destruction des souches de charme par débroussaillants chimiques est imposée aux adjudicataires dans certai- 
nes coupes et les résultats sont excellents sous réserve de tenir la main à l'exploitation et au traitement d'automne.

L'adjudicataire préfère de beaucoup cette mẹ́thode à l'extraction pure et simple exigée auparavant.

Le prix de revient d'une application est de l'ordre de $4000 \mathrm{~F}$ par ha. S'il est nécessaire de parfaire l'opération par une pulvérisation sur rejets d'un an, le coût total est de 8 ooo $\mathrm{F}$ par ha.

A ce prix et sous réserve de la mise au point des méthodes d'emploi, le problème de la conversion clirecte des taillis-sous-futaie est résolu au moins dans la Nièvre où les semenciers sont en nombre suffisant.

\section{Essences des taillis autres que le charme}

Nous n'avons pas spécialement étudié les effets des débroussaillants sur les autres essences que le charme qui sont peu gênantes clans nos conversions.

Cependant, on constate que presque toutes les essences peuvent être détruites par les mêmes procédés.

Une observation particulière doit cependant être faite en ce qui concerne le châtaignier. Cette essence paraît assez résistante à l'application sur souche, mais est très sensible à la pulvérisation même à très faible dose: un essai à $0,3 \%$ de $\mathrm{P} 80$ sur une partie de coupe qui renfermait d'assez nombreuses souches de châtaignier a paru spectaculaire. Il reste à voir par la suite comment se comporteront les souches.

\section{Les recherches à poursuivre}

I $^{\circ}$ Nous avons travaillé jusqu'ici avec des moyens limités, des appareils à dos, une main-d'œuvre souvent réticente surtout après des intoxications passagères.

Sans le dévouement du personnel forestier qui a très souvent exécuté lui-même les opérations, rien n'aurait pu être fait.

Il est donc important de mettre au point par priorité les méthodes d'emploi et l'appareillage.

Le personnel devra être muni: de blouses fermées au col et aux poignets, de gants de caoutchouc et de lunettes protectrices. Il doit être rigoureusement interdit de fumer la cigarette qu'on retire et qu'on reprend avec des mains souillées et qui peut faire aspirer le produit. De même, on ne doit pas se frotter les yeux sans précautions pour ne pas irriter les muqueuses.

Il faut opérer par des jours sans vent violent et jamais à contre vent.

Les appareils à dos doivent avoir toujours des joints en bon état et rles lances pistolet assez longues pour les pulvérisations. 
En fait, les malaises constatés sont dus presque certainement à u11 manque de précautions et à des négligences.

Actuellement, nous faisons mettre au point un pulvérisateur MCV. Cet appareil transporté sur charrette avec une tonne à eau est utilisé en agriculture. La rampe de distribution sera remplacée par deux robinets alimentant deux jets à pistolet en bambou longs de I,50m à $2 \mathrm{~m}$ fixés à l'extrémité de deux tuyaux souples d'une dizaine de mètres.

Cette installation devrait alimenter 2 opérateurs sur 20 à $25 \mathrm{~m}$ de largeur. Le rendement devrait être intéressant et l'emploi moins pénible et moins dangereux que celui des appareils à dos.

Le prix de revient devrait encore s'abaisser sensiblement sur de grandes surfaces.

$2^{\circ}$ Il faudrait étudier les doses d'emploi, car il semble que la pulvérisation bien faite devrait être efficace à des concentrations assez faibles. A I \% la plante est grillée un peu trop vite et le produit n'est sans doute pas complètement absorbé.

Il en est de même pour les traitements des souches si l'on estimait désirable d'obtenir une mortalité échelonnée telle que celle constatée sur les premières placettes d'essai en forêt des Bertranges de I95I à 1956 .

$3^{\circ}$ Il est possible que la pulvérisation, peu, coûteuse si les précautions prises suppriment les accidents, puisse permettre avec les lances pistolet le traitement des, souches au lieu et place du barligeon au pinceau plus long et plus fatigant.

$4^{\circ}$ Il y a lieu de mettre en route des essais étendus de dégagement de plantations résineuses. Les essences résineuses paraissent insensibles à l'action du $\mathrm{P} 80$ dans certaines conditions. Mais, même si le problème était résolu sur le plan théorique, ce qui parait possible, de grosses difficultés surviendront en raison de la situation topographique de la plupart des chantiers: difficultés de transport, d'approvisionnement en eau, d'utilisation de matériel à grand rendement, etc...

Il s'agira donc de savoir à quel prix des solutions pourront être apportées.

Il est vrai que, de plus en plus, la raréfaction de la main-d'ceurre fera passer au second plan la préoccupation des prix de revient comparés entre l'emploi des produits chimiques et du goujard.

$5^{\circ}$ Enfin, les essais faits à l'Inspection de Nevers-Nord ont utilisé des débroussaillants mis au point par le fabricant spécialement et presque sur deniande pour la destruction des essences forestières et particulièrement du charme. Il est fort possible que des perfectionnements puissent être apportés à ces produits ou que d'autres producteurs fabriquent des débroussaillants qui méritent d'être essayés.

Le programme parait donc assez chargé et nécessitera de sérieux 
moyens de tous ordres si l'Administration désire poursuivre dans cette voie.

Pour ce qui nous concerne, la solution à notre propre problème nivernais : la destruction du charme à peu de frais dans les conversions directes, ne demande plus que des mises au point d'ordre pratique, mais il est bien évident que le champ d'action des débroussaillants chimiques devient de plus en plus vaste au fur et à mesure où les dégagements deviennent de plus en plus coûteux et difficiles et la main-d'œuvre plus rare.

\author{
A. Normand, \\ Ingénieur des Eaux et Forèts, \\ Chef de Service à Nevers.
}

A vendre

Collection de la "Revue des Eaux et Forêts "

I896 - 1910 et I9I4 - I929

dont les 7 premiers volumes reliés

Envoyer offres à la Revue forestière 